\title{
Calibração multivariada multiproduto e espectroscopia ultravioleta na determinação da acidez total em bebidas industrializadas a base de soja e néctar de frutas.
}

\author{
Santos, D.A. ${ }^{1}$, Lima, K.P. ${ }^{1}$, Março, P.H. ${ }^{1}$, Valderrama, P. ${ }^{1}$. \\ ${ }^{1}$ Universidade Tecnológica Federal do Paraná (UTFPR), Via Rosalina Maria dos Santos \\ 1233, Caixa Postal 271, CEP 87301-899, Campo Mourão-PR. \\ E-mail para contato: dayane_utfpr87@yahoo.com.br
}

RESUMO - O PLS foi aplicado a espectros ultravioleta para o desenvolvimento de um modelo de calibração multivariada multiproduto para a determinação da acidez total em bebidas industrializadas a base de soja e néctar de frutas. O objetivo foi propor uma metodologia alternativa à potenciometria. Para aquisição dos espectros utilizou-se uma cubeta de quartzo de $1 \mathrm{~mm}$. Um total de 249 amostras foi utilizado, 126 bebidas a base de soja e 123 néctar de frutas. $\mathrm{O}$ algoritmo Kennard-Stone foi empregado na separação das amostras em conjuntos de calibração (185) e validação (64) e as amostras anômalas foram identificadas de acordo com a ASTM E1655-05. O modelo foi construído com 5 variáveis latentes e mostrou exatidão, representada pelos valores dos erros médios quadráticos de calibração (RMSEC) e validação (RMSEP) de 0,049 e $0,045 \mathrm{mg} / 100 \mathrm{~mL}$, respectivamente. Um teste t-pareado mostrou que não existe diferença significativa entre os dois métodos, no nível de confiança de $95 \%$.

\section{INTRODUÇÃO}

É sabido que a determinação da acidez total de bebidas industrializadas a base de soja e de néctar de frutas propicia informações sobre o estado de conservação do produto. E essa acidez, atualmente é quantificada por um método clássico, a potenciometria; entretanto, essa metodologia é relativamente demorada e gera resíduos devido aos reagentes utilizados. Visando tornar o processo de quantificação da acidez total dessas bebidas industrializadas mais eficiente, percebe-se que o modelo de calibração multivariada, em que se aplica o método de mínimos quadrados parciais (PLS) em espectros ultravioleta de tais produtos, pode ser um substituinte da metodologia tradicional aplicada. Isso ocorre devido a robustez do novo método, que além de ser mais rápido e proporcionar resultados eficazes, também evita a geração de resíduos, uma vez que não é necessário preparo de amostras.

Considerando as bebidas industrializadas a base de soja e néctar, pode-se saber qual o estado de conservação do item em questão a partir da acidez total. A acidez dessas substâncias pode ser influenciada por processos de decomposição, sejam eles hidrolise ou fermentação, que alteram quase sempre a concentração dos íons hidrogênio. (VENÂNCIO e MARTINS, 2012 e INSTITUTO ADOLFO LUTZ, 2008). Quanto à maneira de se determinar a acidez é comum realizar a titulação da bebida com uma solução básica de concentração conhecida. Porém, esse processo demanda o uso de reagentes, geram resíduos que podem colocar em risco o analista e o meio ambiente, destroem a amostra, entre outros. 
Conforme as necessidades de redução de desperdício de reagentes, de geração de resíduos, de diminuição nos resultados divergentes e otimização dos processos laboratoriais, de modo geral, observa-se que o uso de uma metodologia alternativa ao modelo clássico de titulação potenciométrica; que alie "avanços computacionais com instrumentação de análises químicas, pode ser uma forma de resolver esse impasse. A quimiometria é uma das áreas da ciência que possibilita o desenvolvimento de novos procedimentos para análise de dados analíticos usando as facilidades existentes para o acoplamento entre os equipamentos de medida e os microcomputadores associadas à facilidade na estocagem de grandes quantidades de dados (LIMA et al., 2008).

De maneira simplificada a quimiometria pode ser definida com a pesquisa e utilização de métodos matemáticos e estatísticos para tratamentos de dados químicos de forma a extrair uma maior quantidade de informações e melhores resultados analíticos (OTTO, 1999). Baseado na quimiometria surgiu a calibração multivariada, que é uma técnica bastante efetiva para determinações simultâneas e na resolução de empecilhos como a superposição de sinais analíticos (OTTO, 1999). A técnica de calibração multivariada se tornou popular com o emprego do método PLS (Partial Least Squares), que é uma metodologia de ajuste linear das variáveis.

Outra dificuldade envolvida nesta determinação da acidez em bebidas é a falta de metodologias alternativas não destrutivas que possam ser utilizadas não apenas pela indústria, técnicas como as espectroscópicas podem ser calibradas a partir de titulações para serem utilizadas com a finalidade de se medir a acidez de néctares de frutas de forma rápida e não destrutiva e portanto, sem geração de resíduos diminuindo custos e aumentando a velocidade das análises. Além disso, para fins industriais a determinação de diferentes produtos no mesmo modelo de calibração multivariada pode ser um ponto muito positivo nas etapas de atualização. A calibração multivariada multiproduto é um tipo de calibração multivariada em que diferentes tipos de amostras (diferentes produtos) são utilizados para na construção do modelo de calibração multivariada, visando à determinação de um mesmo analito de interesse (BERZAGHI et al, 2000). Este tipo de calibração pode ser considerado recente e as vantagens são modelos possivelmente mais robustos, fáceis de atualizar, além da praticidade visando aplicações industriais.

\section{OBJETIVOS}

\subsection{Objetivo Geral:}

Este trabalho tem como objetivo principal a proposição de uma metodologia alternativa para a medição da acidez em sucos de frutas industrializados. Mais especificamente, os estudos sugeridos nesta pesquisa buscam oferecer uma metodologia que apresente vantagens tais como custo relativamente baixo, não utilização de soluções com reagentes químicos e, principalmente, técnicas que além de apresentarem caráter não destrutivo da amostra sejam suficientemente rápidas para serem implementadas na linha de produção industrial.

\section{Objetivos Específicos:}

- Realizar a titulação volumétrica dos sucos de frutas industrializados adquiridos em 
comércio local;

- Adquirir espectros da região Ultravioleta dos sucos de frutas industrializados;

- Construir um modelo de calibração multivariada para os sucos de frutas industrializados;

- Identificar e eliminar possíveis outliers;

-Avaliar os parâmetros de qualidade (RMSEC, RMSEP) dos modelos de calibração multivariada obtidos no estudo;

\section{MATERIAL E MÉTODOS}

As amostras de sucos de frutas industrializados foram adquiridas no comercio de Campo Mourão, Paraná. No total, foram adquiridas 249 amostras de sucos de frutas. Tomando como base a metodologia da Federation International des Producteurs de Jus de Fruit, a acidez dos sucos foram medidas por titulação volumétrica utilizando-se $\mathrm{NaOH}$ na concentração de $0,1 \mathrm{~N}$, padronizado com $\mathrm{HCl} 0,1 \mathrm{~N}$ e indicador de fenolftaleína.

Inicialmente, foram retiradas uma porção de aproximadamente $100 \mathrm{~mL}$ de sucos e colocado em um béquer. Em seguida em uma bureta adicionou a solução de $\mathrm{NaOH} 0,1 \mathrm{~mol} / \mathrm{L}$ padronizados adequadamente na bureta. Os ensaios de titulação foram realizados em triplicata para cada variedade de suco.

Para a realização de medidas espectrais registradas na região Ultravioleta e Visível (UV-Vis) foram avaliadas 3 alíquotas de $1 \mathrm{~mL}$ de cada suco. $\mathrm{O}$ volume foi transferido com pipeta volumétrica para uma cubeta de quartzo de $1 \mathrm{~mm}$ de caminho óptico. Esta cubeta foi utilizada por evitar a etapa de preparo de amostra por diluição, já que aumenta a sensibilidade espectral, melhorando a visualização do perfil espectral por diminuir o número de espécies absorventes.

Após a aquisição dos dados espectrais e dos valores de concentração de ácidos totais por titulação, iniciou-se a parte computacional do trabalho. O software empregado nas análises foi o Matlab 2007b empregando-se o PLS Toolbox 5.2.

Após as análises prévias, foi empregado o método quimiométrico de calibração multivariada para dados de primeira ordem denominado PLS, descrito anteriormente. Para que o PLS possa ser melhor compreendido é necessário fazer uma distinção entre os diferentes tipos de dados. Por exemplo, quando se tem um único valor de absorbância, (absorbância em $520 \mathrm{~nm}$ ) para cada amostra este tipo de dado é dito univariado ou de ordem zero. Quando se tem um espectro como resposta para cada amostra tem-se um dado de primeira ordem. Já no caso de se obter uma superfície de resposta para cada amostra têm-se dados de segunda ordem (espectros com o tempo) (VALDERRAMA, et al., 2009).

Os espectros UV foram organizados no formato de uma matriz, em que cada linha da matriz $\mathbf{X}$ corresponde a um espectro e cada coluna corresponde à absorbância nos diferentes comprimentos de onda. Na figura 2 é possível observar a organização dos espectros no formato da matriz. A matriz $\mathbf{X}$ é então correlacionada a um vetor $\mathbf{y}$ que contem os valores de acidez determinados por titulação. A correlação entre $\mathbf{X}$ e $\mathbf{y}$ é realizada através do método de 
calibração multivariada PLS.

\section{RESULTADOS E DISCUSSÃO}

A Figura 1 apresenta a região utilizada dos espectros na região do UV utilizada na construção do modelo de calibração multivariada para bebidas analisadas a base de néctar (A) e a base de soja (B). Na Figura 1(C), os espectros de ambas as bebidas são mostrados de forma sobreposta.
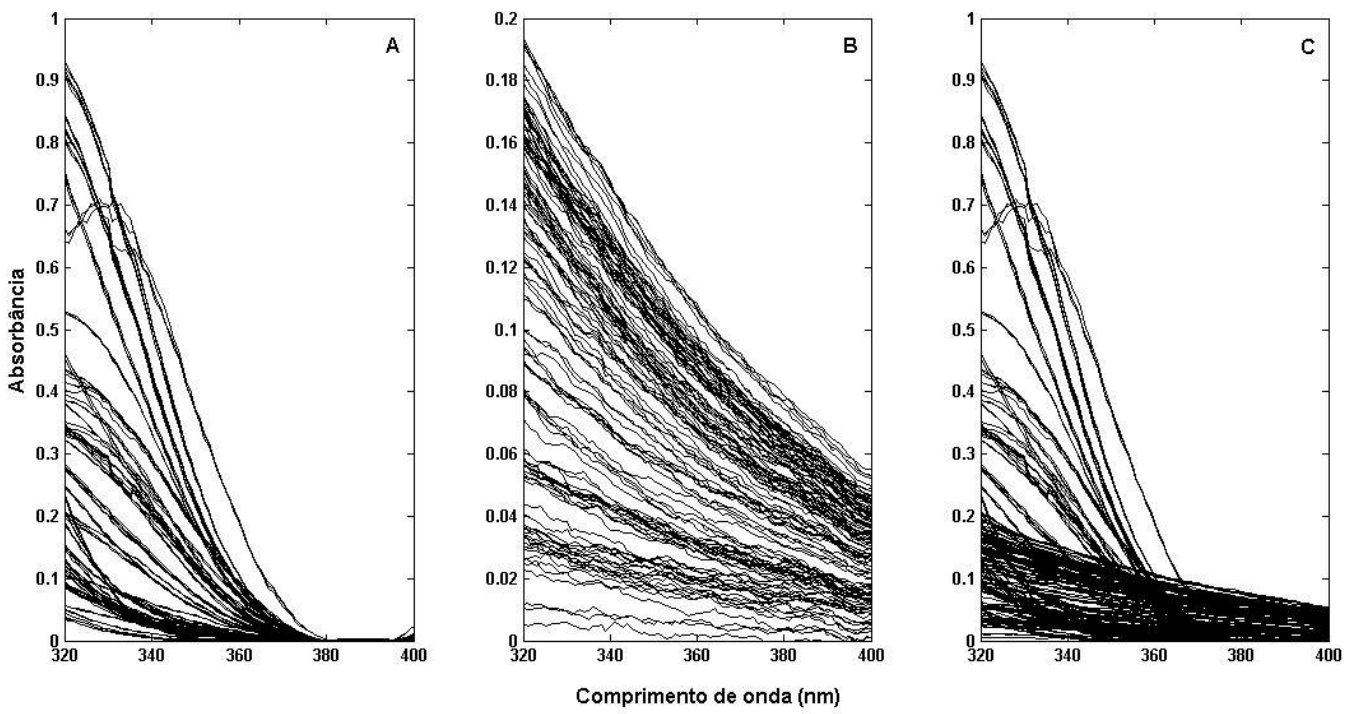

Figura 1. Espectros UV para as bebidas: (A) néctar, (B) a base de soja, (C) néctar e bebidas a base de soja conjuntamente.

A região entre 320 a $400 \mathrm{~nm}$ foi utilizada na construção do modelo de calibração multivariada. Nesta região espectral os valores de absorbância foram inferiores a uma unidade e, portanto, são proporcionais à concentração, conforme previsto pela Lei de Beer e esta região tem sido proposta na literatura para modelos de calibração multivariada através do método PLS e espetroscopia UV (BUSCH et al, 2003, BUSCH et al, 2004, FAKAYODE et $a l, 2005)$.

Para construção dos modelos de calibração as amostras foram separadas entre conjunto de calibração e validação através do algoritmo de Kennard-Stone (KENNARD e STONE, 1969). Este algoritmo seleciona as amostras com base em suas distâncias: a primeira amostra selecionada é a que apresenta a maior distância em relação à amostra média enquanto a segunda amostra a ser selecionada será a que apresentar maior distância em relação à primeira amostra selecionada. A próxima amostra a ser selecionada apresentará maior distância em relação à última amostra selecionada, e assim sucessivamente até atingir o número de amostras desejadas. Esse algoritmo é aplicado para realizar a seleção das amostras que irão compor o conjunto de calibração, uma vez que procede a seleção das amostras de maior variabilidade, ou seja, as amostras mais "externas" do conjunto total. Assim, cada conjunto de calibração foi inicialmente composto por 185 amostras enquanto o conjunto no conjunto de 
validação foram utilizadas 64 amostras.

Para a construção dos modelos, os espectros foram centrados na média e o número de variáveis latentes foi determinado através dos resultados da raiz quadrada do erro quadrático médio de validação cruzada (RMSECV) para as amostras de calibração, obtido por validação cruzada do tipo "leave-on-out". Nesse método de validação interna o modelo é construído e faz-se a previsão de uma das amostras de calibração que foi 'deixada de fora' no momento da construção do modelo. Esse procedimento é repetido até que todas as amostras do conjunto de calibração tenham sido previstas. Para o modelo de quantificação da acidez nas bebidas foram necessárias um total de 5 variáveis latentes e as amostras anômalas foram avaliadas com base no resíduo espectral, resíduo na variável dependente e leverage para as amostras de calibração, enquanto para as amostras de validação as anomalias foram identificadas com base no resíduo espectral e no leverage. Esses testes são descritos pela norma ASTM E1655-05.

Os resultados dos parâmetros de qualidade do modelo desenvolvido são representados pelos valores do erro médio quadrático de calibração (RMSEC) e previsão (RMSEP). Os valores de exatidão são representados RMSEC e RMSEP e apresentaram valores 0,049 e $0,045 \mathrm{mg} / 100 \mathrm{~mL}$, esses resultados revelam que os valores estimados pelos modelos multivariados apresentaram concordância com os resultados esperados. Entretanto, os valores de RMSEP e RMSEC são parâmetros globais que incorporam tanto erros sistemáticos quanto aleatórios. Sendo assim, um melhor indicador de exatidão é a regressão entre os valores de referência e os valores estimados pelo modelo, e os valores da inclinação e do intercepto, apresentados na Figura 2.

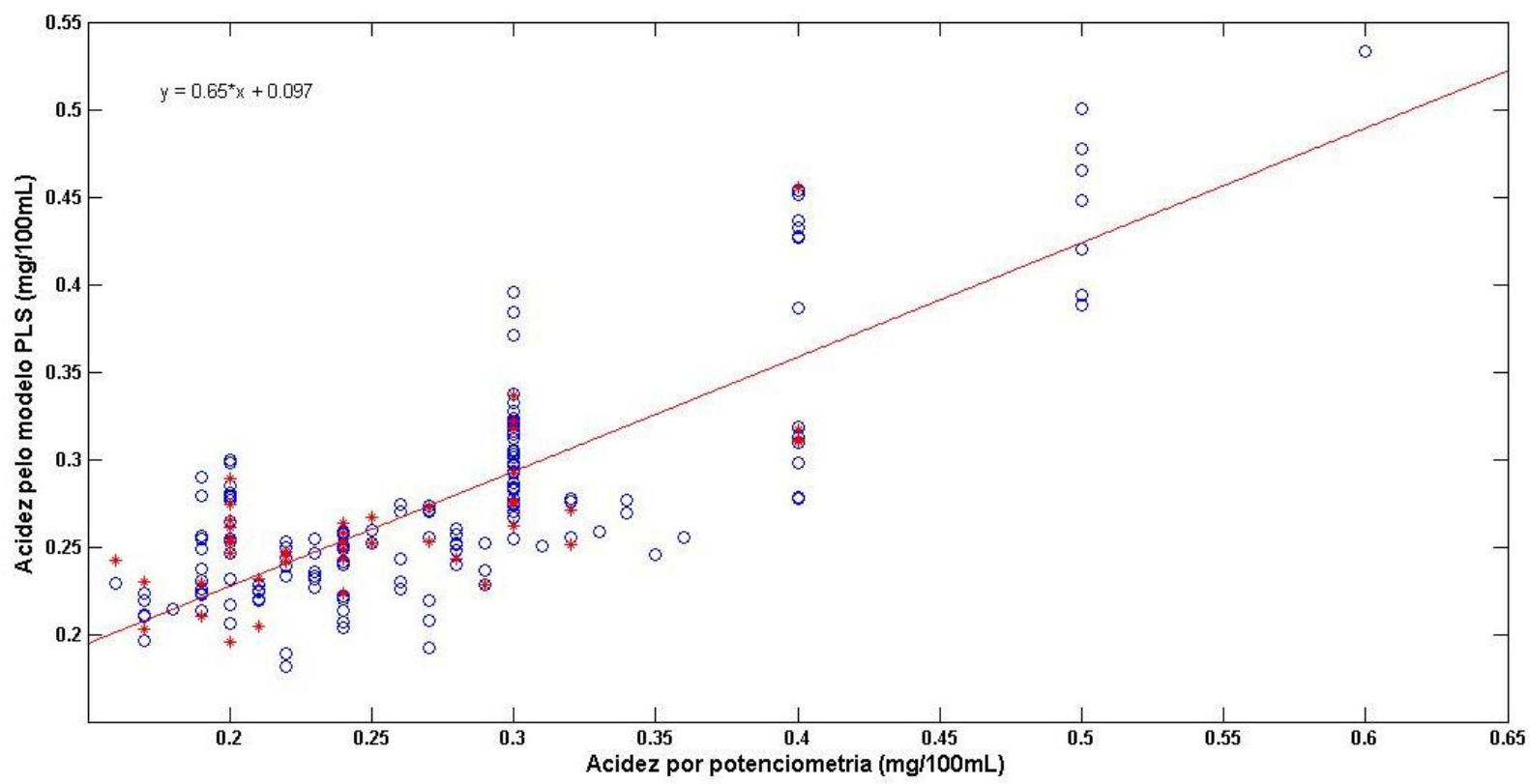

Figura 2. Ajuste do modelo PLS para determinação da acidez. (o) amostras de calibração. (*) amostras de validação.

O coeficiente de determinação para o ajuste foi da ordem de 0,7. Apesar de parecerem valores muito baixos, resultados de coeficiente de correlação dessa ordem já foram reportados antes na literatura quando o método de referência, na calibração multivariada, é a titulação 
(VALDERRAMA et al., 2007; VALDERRAMA et al., 2007; FERREIRA et al., 2013).

Os resultados alcançados para determinação da acidez em néctar e bebidas a base de soja através da espectroscopia na região do UV e calibração multivariada multiproduto estão correlacionados de tal forma a sugerir que o método se apresenta como muito promissor. Um teste de significância, teste t-pareado, foi utilizado para comparar os dois métodos: modelo PLS e titulação potenciométrica. O teste t-pareado, que indica se existem diferenças significativas entre os métodos utilizados, apresentou um valor para tcalculado de 0,286, enquanto o valor de ttabelado é de 2,131 para 95\% de confiança. Como o valor de tcalculado é menor que o valor de ttabelado, isso indica que não existem diferenças significativas entre a titulação de neutralização e o modelo PLS na determinação da acidez das bebidas, no nível de significância considerado.

\section{CONCLUSÃO}

O desenvolvimento deste trabalho mostrou a possibilidade da utilização de métodos espectroscópicos associados à métodos quimiométricos para a determinação da acidez em bebidas industrializadas. Os modelos construídos por calibração de primeira ordem utilizando o método PLS mostraram resultados satisfatórios para essa determinação. Um teste de significância foi aplicado aos resultados obtidos pelo modelo PLS e os resultados obtidos pela titulação potenciométrica mostrando que, no nível de confiança de 95\%, os métodos não apresentam diferença significativa.

\section{REFERÊNCIAS}

ANNUAL BOOK OF ASTM STANDARDS. Standards practices for infrared multivariate quantitative analysis. E1655-05. West Conshohocken, Pennsylvania, USA: ASTM international, 2012.

Berzaghi, P.; Shenk, J. S.; Westerhaus, M.O.; Prediction with near infrared multi-product databases. J. Near Infrared Spectrosc. v. 8 p. 1-9, 2000.

Busch, K. W.; Swamidoss, I. M.; Fakayode, S. O.; Busch, M. A.; Determination of the enantiomeric composition of some molecules of pharmaceutical interest by chemometric analysis of the UV spectra of cyclodextrin guest-host complexes. Analytica Chimica Acta, 525(1), 53-62, 2004.

Busch, K.W.; Swamidoss, I.M.; Fakayode, S.O.; Busch, M.; Determination of the enantiomeric composition of guest molecules by chemometric analysis of the UV-Visible spectra of cyclodextrin guest-host complexes. Journal of American Chemical Society, v. 125(7), p. 16901691, 2003.

Fakayode, S.O.; Busch, M.A.; Bellert, D.J.; Busch, K.W.; Determination of the enantiomeric composition of phenylalanine samples by chemometric analysis of the fluorescence spectra of 
cyclodextrin guest-host complexes. Analyst, v. 130(2), p. 233-241, 2005.

FERREIRA, D. S.; PALLONE, J. A. L.; POPPI, R.J.; Fourier transform near-infrared spectroscopy (FT-NIRS) application to estimate Brazilian soybean [Glycine max (L.) Merril] composition, Food Research International. v. 51, p.53-58, 2013.

INSTITUTO ADOLFO LUTZ. Métodos físico-químicos para análise de alimentos, $2008 .$.

Kennard, R. W.; Stone, L. A.; Computer aided desing of experiments, Technometrics. v. 11(1), p. 137-148, 1969.

Otto, M.; Chemometrics. Weinheim: Wiley, 1999.

VALDERRAMA, P.; BRAGA, J. W. B.; POPPI, R. J.; Estado da arte de figuras de mérito em calibração multivariada, Química Nova, 32, 1278-1287, 2009.

Valderrama, P.; Braga, J.W.B.; Poppi, R. J.; Validation of multivariate calibration models in the determination of sugar cane quality parameters by near infrared spectroscopy. Journal of the Brazilian Chemical Society, v. 18(2), p. 259-266, 2007.

Valderrama, P.; Braga, J.W.B.; Poppi, R. J.; Variable selection, outlier detection, and figures of merit in a partial least-squares regression multivariate calibration model. A case study for the determination of quality parameters in the alcohol industry by near-infrared spectroscopy. Journal of Agricultural and Food Chemistry, v. 55(21), p.8331-8338, 2007.

VENÂNCIO, A.A., MARTINS, O.A. Análise química de diferentes marcas de néctares e suco de laranja comercializada na cidade de Cerqueira César. Revista Eletrônica de Educação e Ciência (REEC). v. 02, p. 45-50, 2012. 